\title{
Comprehensive analysis of metabolism-related IncRNAs related to the progression and prognosis in osteosarcoma from TCGA
}

\author{
Xingyin Chen ${ }^{1}$, Zhengyun Ye ${ }^{1}$, Pan Lou ${ }^{1}$, Wei Liu ${ }^{1}$ and Ying Liü ${ }^{2 *}$
}

\begin{abstract}
Background: Osteosarcoma is one of the most common malignant neoplasms in children and adolescents. Studies have shown that metabolism-related pathways are vital for the development and metastasis of osteosarcoma. Long non-coding RNA (IncRNA) plays a key role in the occurrence and progression of cancer in a variety of ways. However, the detailed molecular mechanisms of metabolism-related IncRNA in osteosarcoma remain to be deeply elucidated.

Methods: In this study, all metabolism-related mRNAs and IncRNAs in osteosarcoma were extracted and identified based on transcriptomic data from the TCGA database. Usingsurvival analysis, univariate and multivariate independent prognostic analysis, gene set enrichment analysis, and nomogram, a prognostic signature with metabolic IncRNAs as prognostic factors was constructed.

Results: Nine prognostic factors included IncRNA AC009779.2, IncRNA AL591895.1, IncRNA AC026271.3, IncRNA LPP-AS2, IncRNA LINC01857, IncRNA AP005264.1, IncRNA LINC02454, IncRNA AL133338.1, and IncRNA AC135178.5, respectively. Survival analysis indicated that alterations of specific IncRNA expression were strongly correlated with poor prognosis in osteosarcoma. Univariate and multivariate independent prognostic analysis showed that the prognostic signature had a good independent predictive ability for patient survival. The results of GSEA suggested that these predictors may be involved in the metabolism of certain substances or energy in cancer. The nomogram was further drawn for clinical guidance and assistance in clinical decision-making.

Conclusions: This study identified multiple metabolism-related IncRNAs, which may be novel therapeutic targets for osteosarcoma, and contributed to better explore the specific metabolic regulatory mechanisms of IncRNA in osteosarcoma.
\end{abstract}

Keywords: Osteosarcoma, Metabolism-related IncRNA, Prognosis signature, GSEA

\section{Introduction}

Osteosarcoma, one of the most common Sarcoma, is usually diagnosed during childhood and adolescence [1]. Due to always occurring in the metaphysis of long bones

*Correspondence: rainly.1988@163.com

${ }^{2}$ Department of Gastroenterology, The First People's Hospital of Jingmen, Xiangshan Avenue 168, Jingmen 448000, Hubei, China

Full list of author information is available at the end of the article and progressing rapidly, it often results in inconvenient or even restricted movement of patients [1]. Although effective progress has been made in current amputation and chemotherapy, patient prognosis is far from satisfactory, with $20 \%$ of patients metastasized at diagnosis [2]. Patients with metastatic osteosarcoma have a lower survival rate [3], which demands a better understanding of the mechanisms underlying osteosarcoma progression original author(s) and the source, provide a link to the Creative Commons licence, and indicate if changes were made. The images or other third party material in this article are included in the article's Creative Commons licence, unless indicated otherwise in a credit line to the material. If material is not included in the article's Creative Commons licence and your intended use is not permitted by statutory regulation or exceeds the permitted use, you will need to obtain permission directly from the copyright holder. To view a copy of this licence, visit http://creativecommons.org/licenses/by/4.0/. The Creative Commons Public Domain Dedication waiver (http://creativeco mmons.org/publicdomain/zero/1.0/) applies to the data made available in this article, unless otherwise stated in a credit line to the data. 
and metastasis. Therefore, it is particularly important to explore new therapeutic targets and accurate prognostic markers. Long non-coding RNA (lncRNA) has been shown to play an important regulatory role in the occurrence, development, and metastasis of all kinds of cancers [4-6]. Recently, the research on the abnormal expression of lncRNA in osteosarcoma has become ever more in-depth $[7,8]$. On the other hand, growth of bone tissue is inextricably linked to bone metabolism [9]. Abnormal bone metabolism can lead to loss of control of bone growth or abnormal differentiation, which further results in osteoma or osteosarcoma [9]. Despite many therapeutic targets and prognostic markers for osteosarcoma have been reported, such as immunomodulation, tumor infiltrating immune cells could be used as a prognosis factor in osteosarcoma patients [10], Niu et. al had found that EGR1, CXCL10, MYC and CXCR4 could be regarded as potential biomarker of osteosarcoma [11]. Few reports show that lncRNA affects osteosarcoma by regulating the metabolic process [12], and the roles of most lncRNAs in regulating the metabolism of osteosarcoma remain unclear.

In this study, gene expression data and clinical information were available from The Cancer Genome Atlas (TCGA) database. Metabolic genes and related lncRNAs were further extracted. Nine Metabolism-related lncRNAs (MRlncRNAs) associated with high prognosis were finally obtained with various bioinformatics analysis methods. We further constructed and verified a prognostic signature of osteosarcoma. This study explains the possible metabolic epigenetic mechanism and provides new therapeutic targets of osteosarcoma.

\section{Materials and methods}

\section{Data downloading and processing}

All transcriptions data of osteosarcoma samples were downloaded from the TCGA database [13]. There was a total of 265 samples. After removing duplicated and extremely low expression genes, the mRNA and lncRNA matrixes were extracted, respectively. The c2.cp.kegg. v7.2.Symbols.Gmt from the Gene Set Enrichment Analysis (GSEA) database was downloaded [14], a total of 940 related genes in metabolic pathways were extracted. Furthermore, the intersection of the extracted mRNA matrix was taken to obtain the expression data of 935 metabolic genes.

\section{Preliminary Screening of MRIncRNA}

By using the correlation test in R 4.0.3, 335 lncRNAs that highly related to metabolic genes (corFilter $=0.6$, pvalueFilter $=0.001$ ) were acquired. 15 lncRNAs significantly related to prognosis were obtained by combining with year of survival through Kaplan-Meier survival $(P<0.05)$ analysis and univariable Cox regression survival $(P<0.05)$ analysis.

\section{Construction and evaluation of prognostic signature}

We used "glmnet" package in R 4.0.3 to perform Least Absolute Shrinkage and Selection Operator (LASSO) regression analysis, and further used the multivariable Cox regression analysis to obtain prognosis-related metabolic lncRNA. These factors were used to construct a prognostic signature. Then, the risk score signature for prognostic metabolism of lncRNA was established according to the following formula: Risk Score (RS) $=\Sigma$ $\beta_{(M R \ln c R N A)} \times \operatorname{Exp}_{(M R \ln c R N A)} \cdot \beta$ represents the coefficient of the multivariable Cox regression analysis. $\operatorname{Exp}_{\text {(MRIncRNA) }}$ means the individual prognostic metabolic lncRNA expression value. The risk score of each osteosarcoma patient was calculated accurately, then, all patients were divided into high-risk and low-risk groups through the median risk score. In addition, a survival curve was drawn to assess the difference in survival between the two groups by utilizing the "survival" package in R 4.0.3.

\section{Metabolism-related mRNA-IncRNA network construction} Nine hundred thirty five metabolic genes and $335 \mathrm{MRl}-$ ncRNAs extracted from the TCGA database were utilized to construct the co-expression network. Similarly, we also constructed the network of lncRNAs and related metabolic genes with prognostic signatures. The visual co-expression network was produced by Cytoscape 3.7.2.

\section{Independent prognosis and clinical correlation analysis}

Univariate and multivariate Cox regression analyses were applied to determine independent clinical prognostic factors, that is, whether individual lncRNA has a potential impact on certain clinical features or not. $P$ value $<0.05$ was considered statistically significant.

\section{Survival analysis}

By using the $\mathrm{R}$ "survival" package, the risk model was analyzed and evaluated whether the high- or low-risk group was related to survival. Similarly, each prognostic factor was divided into high- and low-expression groups according to the median expression value, then the survival curve was drawn. $P$ value $<0.05$ was statistically significant.

\section{Nomogram drawing and evaluation}

The nomogram was drawn with the known clinicopathological data and the risk score. Firstly, the receiver operator characteristic (ROC) curve was used to evaluate its representativeness. Secondly, the $C$ index was used to evaluate its predictive ability. In addition, the calibration curves of 1-year, 3-year and 5-year survival rates were 
established to evaluate the accuracy of the nomogram. Finally, to reduce the dimensionality of the data and figure out whether or not an individual lncRNA could distinguish patients' risk groups, we performed principal component analysis (PCA).

\section{Result}

The entire research process is shown in Fig. 1.

\section{Screening of MRIncRNAs}

By downloading the transcriptions data of osteosarcoma patients, the mRNA and lncRNA matrixes were extracted, respectively. A total of 940 genes related to metabolic pathways were acquired from the GSEA database. The expression data of 935 metabolic genes were extracted accurately. And 335 lncRNAs, which are closely correlated with metabolic genes, were obtained by correlation test in the R. These lncRNAs. A network of 935 metabolic genes and 335 related lncRNA was constructed and visualized by Cytoscape (Fig. 2). Most mRNAs and lncRNAs in this network have a one-to-many or manyto-one relationship, which initially reveals the complexity and extensiveness of lncRNAs network regulation. After incorporating the complete prognostic information, we utilized Kaplan-Meier survival analysis and univariate Cox regression survival analysis to obtain 15 lncRNAs significantly related to the prognosis (Supplementary Table 1).

\section{Construction and evaluation of risk signature}

To further reduce and optimize the signature, the $R$ "glmnet" package was used to perform LASSO regression analysis, and the model showed best fits when $\lambda=-3$ and $\mathrm{N}=9$ (Fig. $3 \mathrm{a}-\mathrm{b}$ ). We further used the multivariate Cox regression analysis to obtain nine prognostic metabolic lncRNA and drew a forest map (Fig. 3c). It reveals that lncRNA AC026271.3, lncRNA LINC02454, lncRNA AL133338.1, and lncRNA AC135178.5 are high-risk factors for patient survival time and status, which implies that these four lncRNAs may act as carcinogens. Since lncRNA AC009779.2 is a low-risk factor, it is very likely to become a protective factor. Then we used these 9 factors to construct a prognostic risk signature:

Risk Score (RS) = AC009779.2*-0.08566 + AL591895.1* $0.03249+\mathrm{AC} 026271.3^{*} 0.116683+\mathrm{LPP}$ AS2*-0.22239+ LINC01857*-0.35896 + AP005264.1*$0.21311+$ LINC02454*0.065331+AL133338 $0.1^{*} 0.12001$ $6+\mathrm{AC} 135178.5 * 0.134252$.

According to this formula, regarding the median risk score as the grouping standard, we can accurately calculate the risk score of each osteosarcoma patient. Then all patients were subdivided into high- or low-risk groups. The survival difference between the two groups was evaluated by drawing a survival curve (Fig. 3d). Patients in the low-risk group were under a higher survival rate while patients in the high risk group had a worse survival rate $(P<0.001)$. In addition, we drew a risk curve and a heat map of the expression of the nine lncRNAs in all samples based on the constructed signatures (Fig. 3eg). This signature can accurately distinguish high-risk patients from low-risk groups through different risk score and survival time.

\section{Network construction of IncRNAs and related mRNAs}

In the next step, we also constructed a grid of nine lncRNAs and their related metabolic genes. Figure 4a shows a Sankey diagram constructed based on co-expression relationships. The left part shows related metabolic genes. The middle part indicates lncRNAs that are highly related to metabolic genes, and the right part represents prognostic types of lncRNAs. For example, LINC01857 may have a co-expression relationship with DGKA, PLCG2, and PLA2G2D, which means that some interactions or mutual regulation mechanisms exist between them. In addition, lncRNA LINC01857 shows a protective role, suggesting that it may act as a tumor suppressor gene in the development of cancer. Similarly, results of Cytoscape 3.7.2 (Fig. 4c) intuitively reveal the mRNA-IncRNA coexpression relationships.

\section{Independent prognosis and clinical correlation analysis}

Next, to determine whether it applies to guide clinical decision-making or assist in evaluating the prognosis, we verified the independent prognostic capacity of the prognostic signature constructed above by using the univariate Cox regression analysis respectively. In the univariate Cox regression analysis, the hazard ratio of the risk score is $1.538(1.313-1.802)$ and is statistically significant $(P<0.001)$ (Fig. 4b). Similarly, in the multivariate regression analysis, the hazard ratio of the risk score is $1.549(1.286-1.865)$ and reveals a significant statistical significance $(P<0.001)$ (Fig. $4 d)$. Furthermore, the multiindex ROC curve showed that the risk score has better diagnostic and recognition capabilities for patients with osteosarcoma (AUC $=0.861$ ) than other clinical features (Fig. 4e). In addition, we further analyzed whether a single lncRNA has a potential impact on certain clinical characteristics (Fig. 5). For example, as shown in Fig. 5a, lncRNA AL591895, lncRNA LINC02454, and IncRNA AL133338.1 show definite differences in tumor populations over and under 65 years of age and younger $(P$ value $<0.05$ ). Similarly, lncRNA AC026271.3 and lncRNA AL133338.1 also show partial expression differences for patients of different genders $(P$ value $<0.05)$ in Fig. $5 \mathrm{~b}$. LncRNA AC009779.2 appears to be related to cancer metastasis $(P$ value $<0.01)$ in Fig. 5c. 


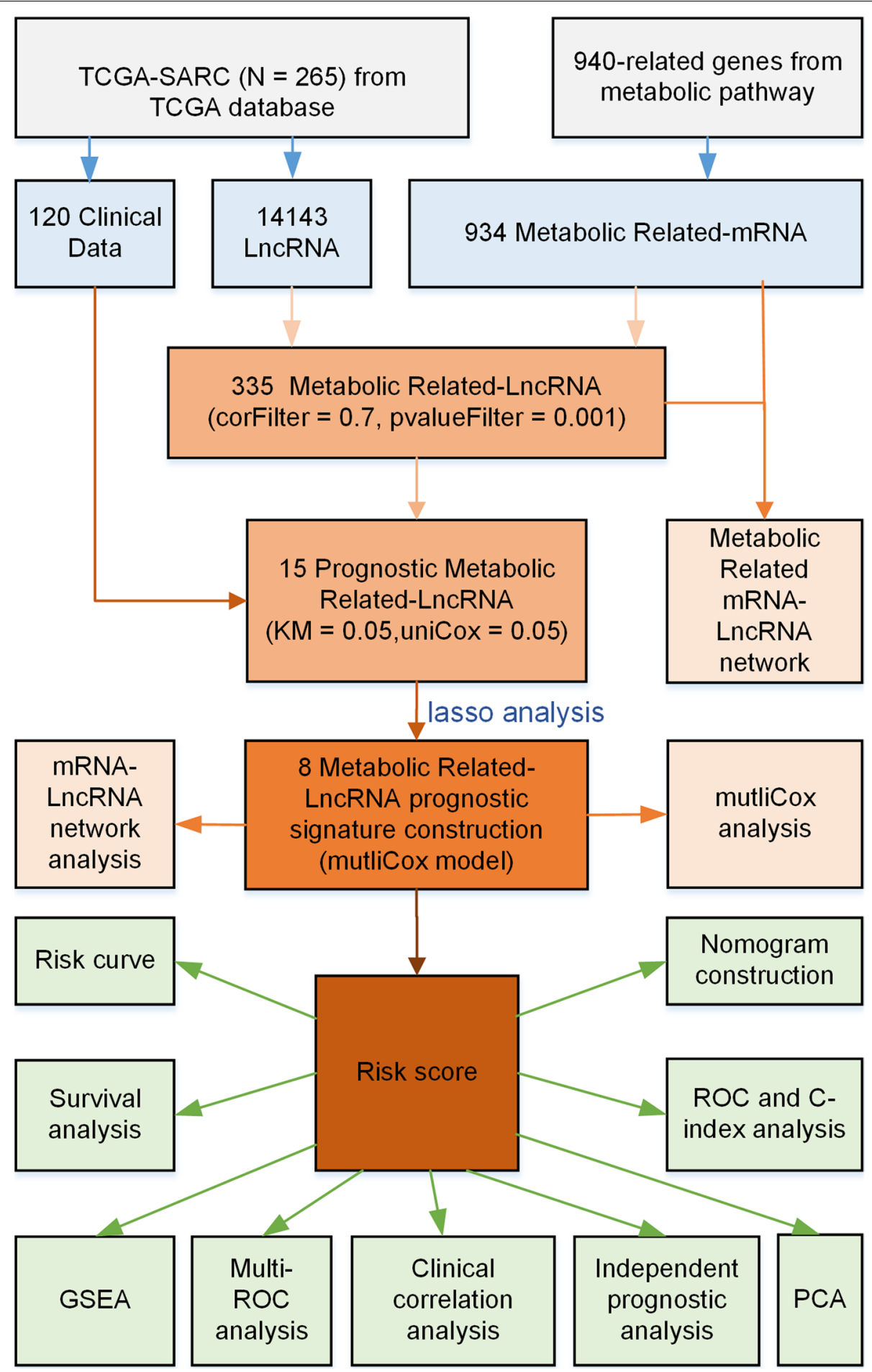

Fig. 1 Flow diagram of entire data analysis

\section{Prognostic survival analysis of MRIncRNA}

To further evaluate the independent prognostic capacity of single lncRNA in the signature, we divided each lncRNA into high- and low-expression groups according to the median expression value and drew the survival curve (Fig. 6). It reveals that all nine prognostic factors are statistically significant, and patients with two different prognoses can 

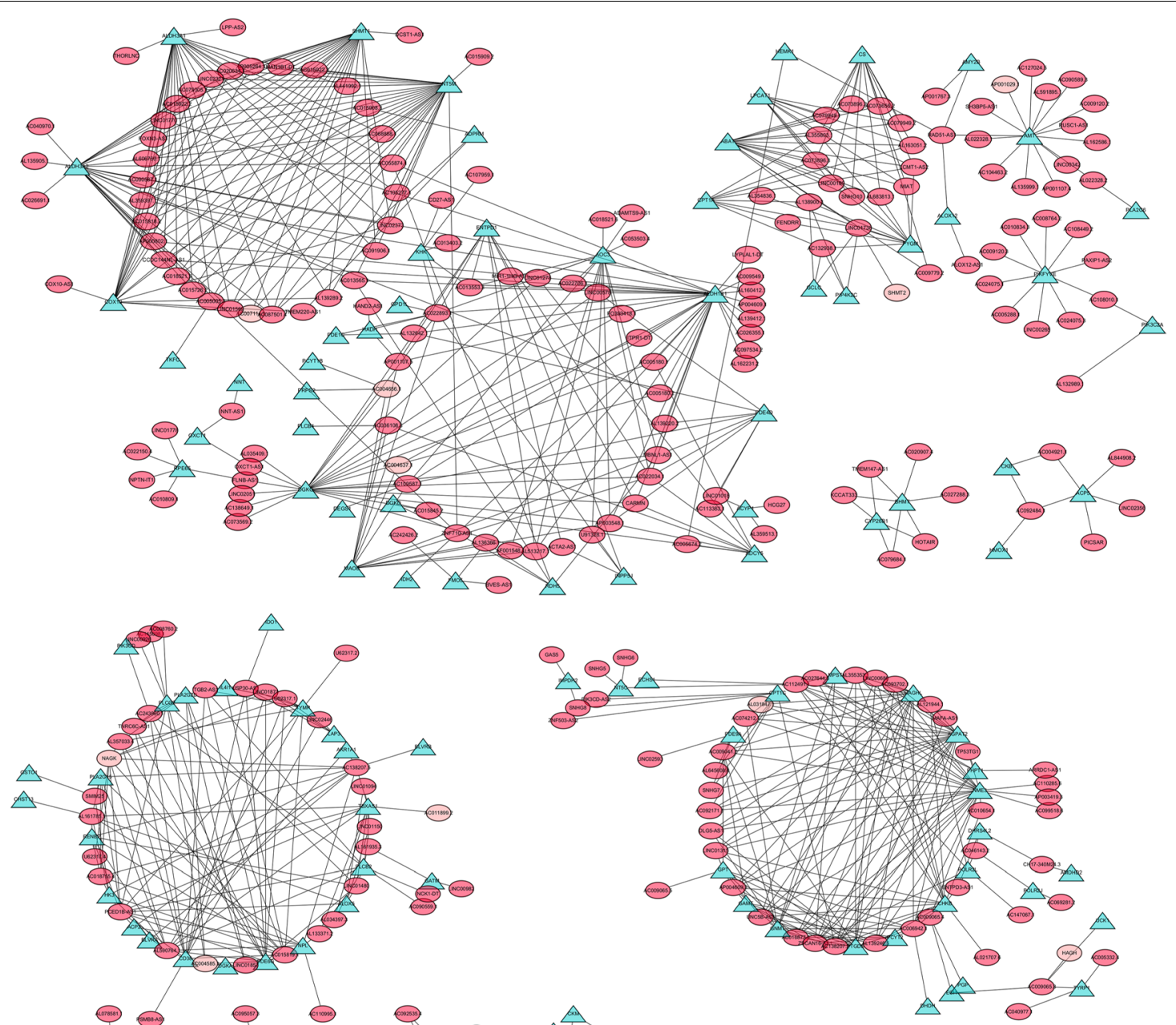

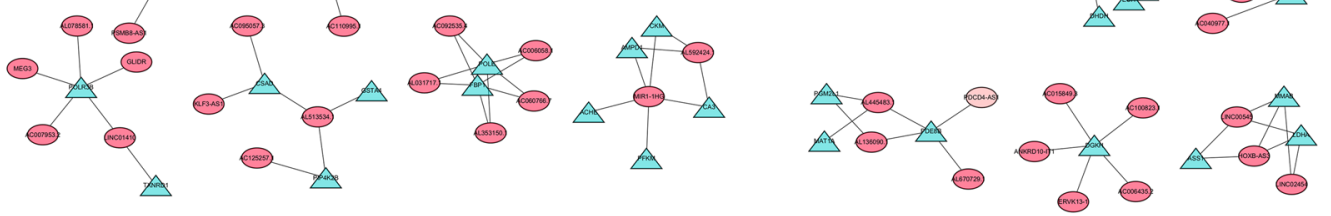

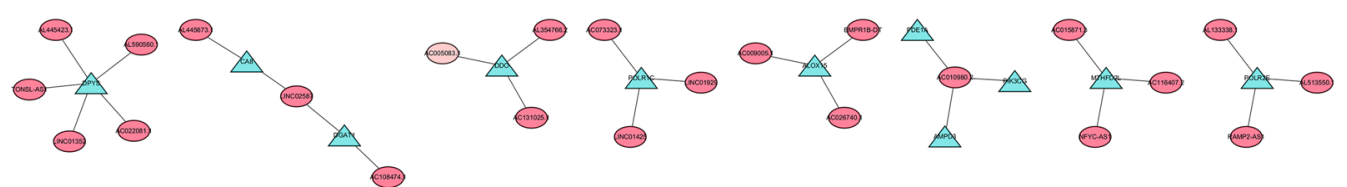

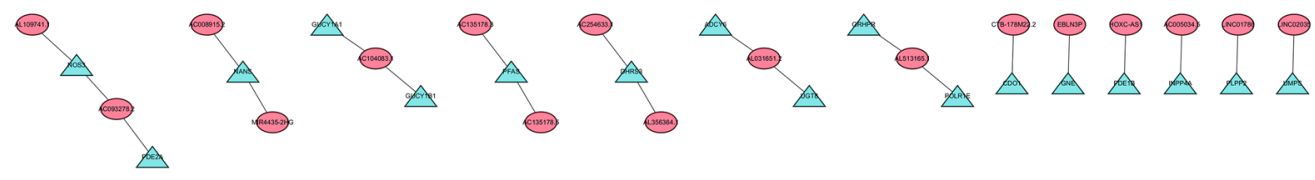

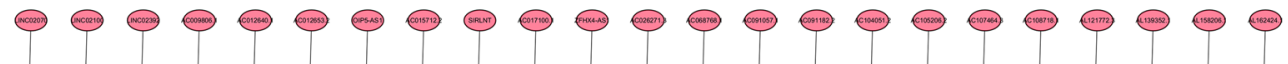

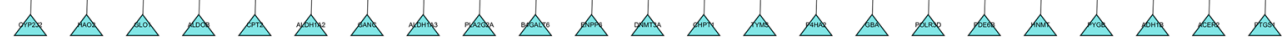

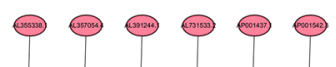

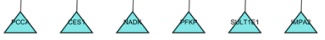

LncRNA

$\triangle$ mRNA

Fig. 2 mRNA-IncRNA network of 935 metabolic genes and 335 metabolic-related IncRNAs 


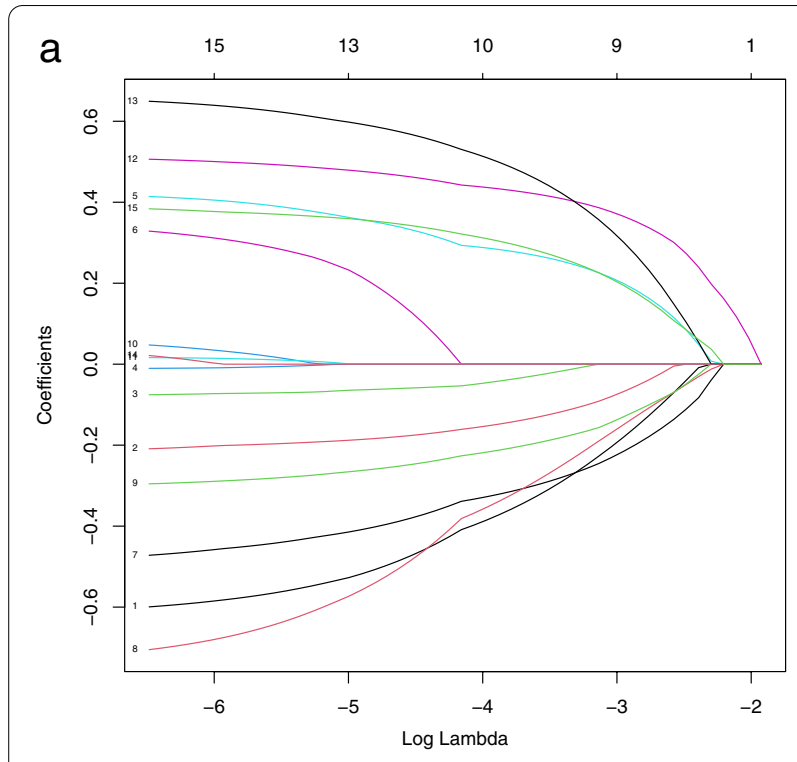

C

Hazard ratio

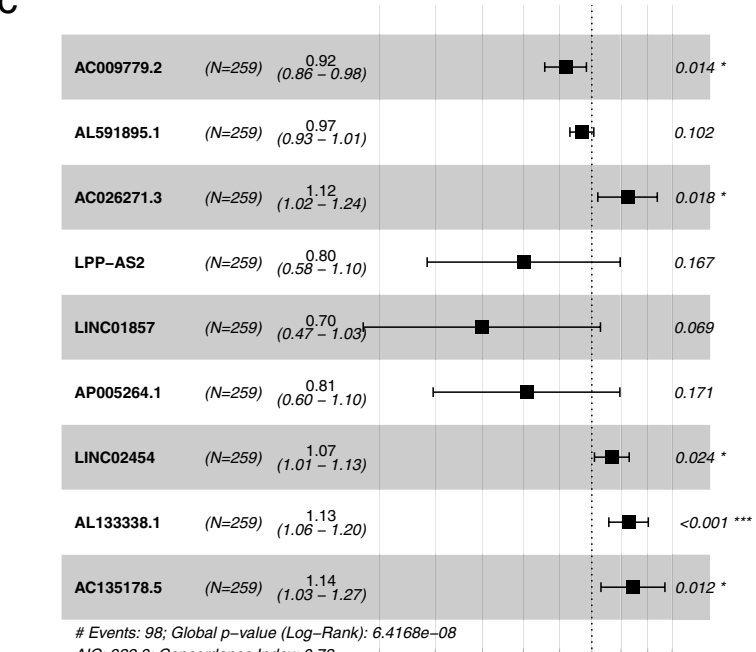

e

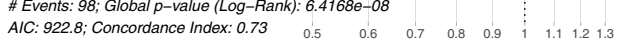

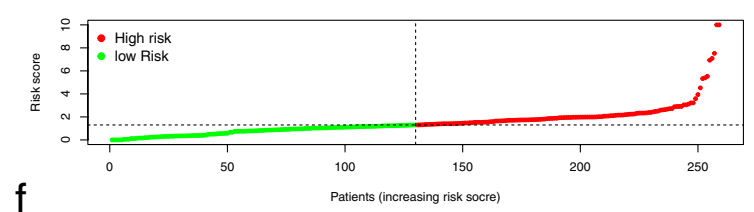

$f$

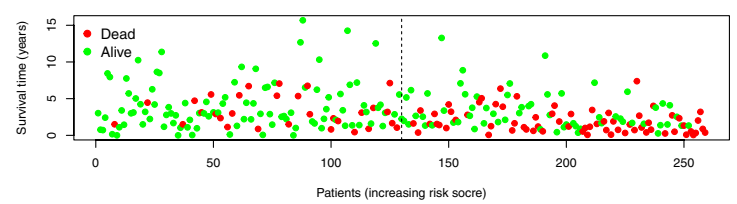

$\begin{array}{lllllllllllllllll}15 & 15 & 14 & 14 & 14 & 13 & 11 & 11 & 11 & 10 & 10 & 10 & 9 & 9 & 9 & 5 & 1\end{array}$

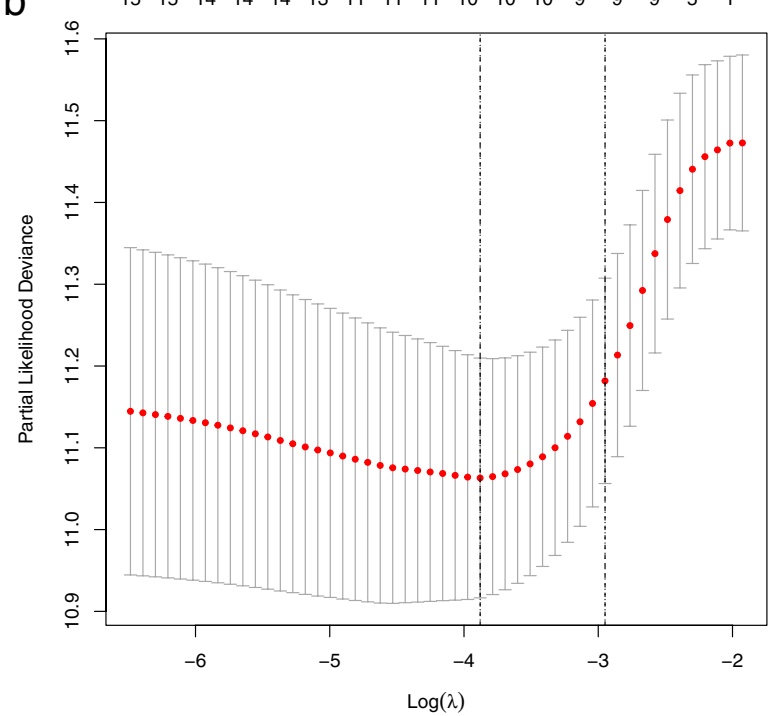

d

Survival curve $(p=1.238 \mathrm{e}-08)$
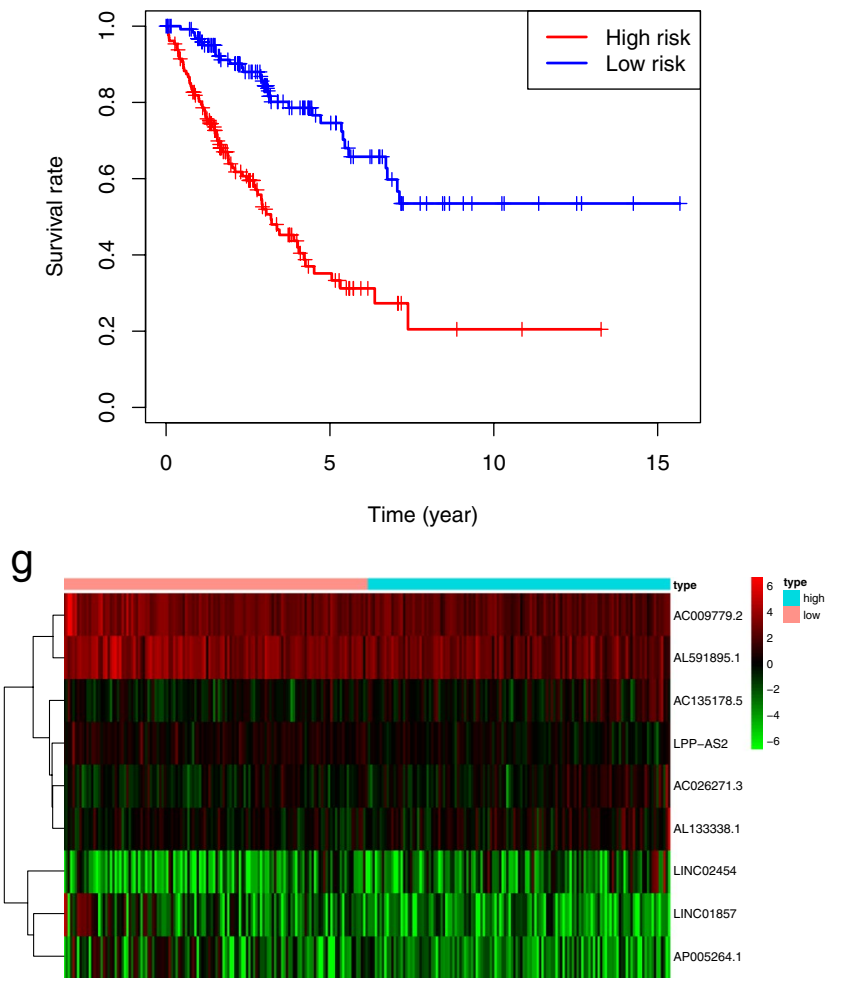

Fig. 3 The prognostic signature was established and validated based on nine prognostic metabolic IncRNAs. a LASSO coefficient profiles of the IncRNAs associated with the metabolism of osteosarcoma, $\mathbf{b}$ Partial likelihood deviance is plotted versus log $(\lambda)$. c The forest map shows multivariable COX analysis of prognostic signatures. $\mathbf{d}$ The survival curve indicates the difference between high and low risk group. e The risk curves were reordered based on the risk score of each patient. f The scatter plot displayed the overall survival in osteosarcoma patients. $\mathbf{g}$ The heatmap revealed the expression levels of metabolic IncRNAs 


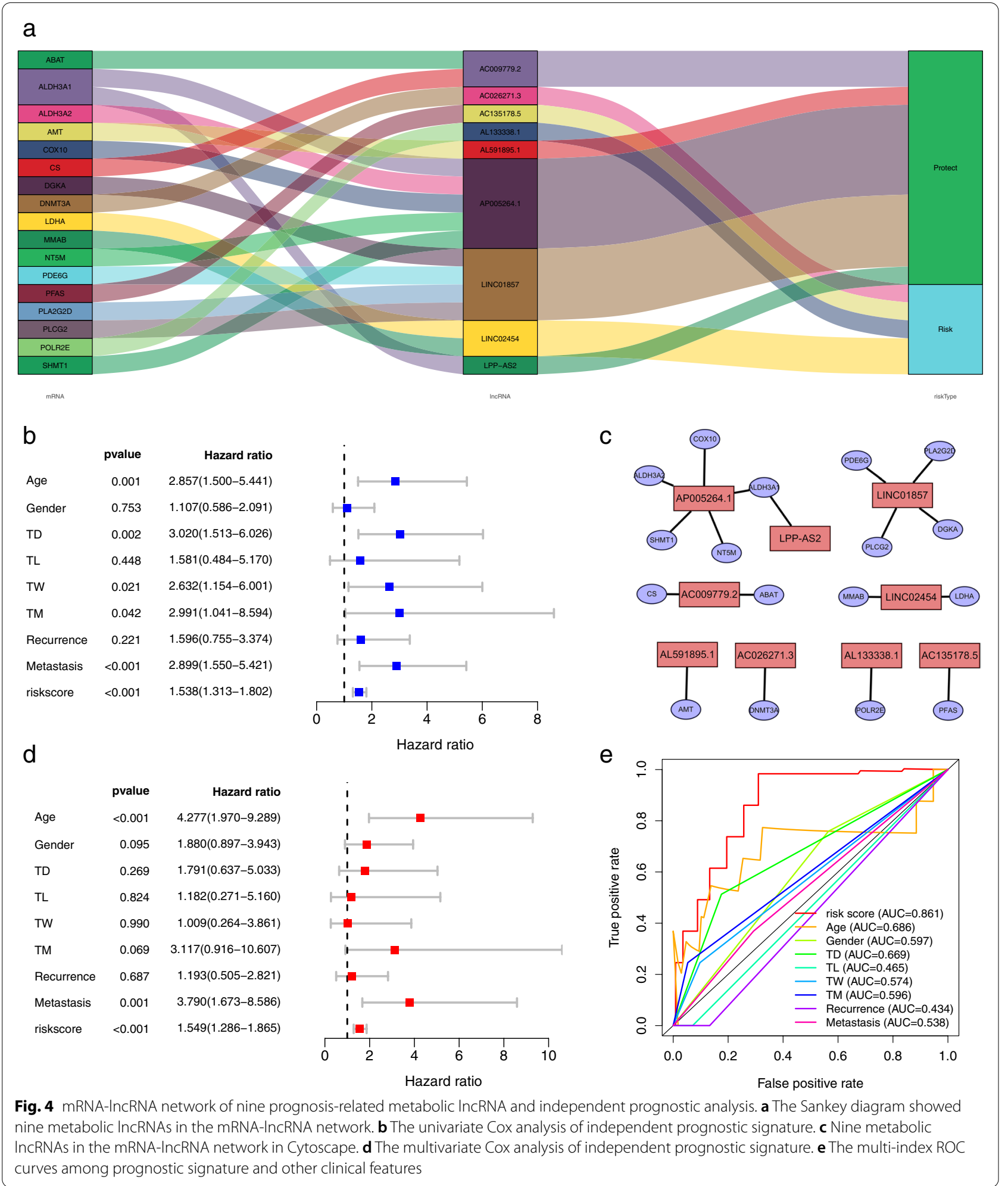

be distinguished. It further illustrates that they may play potential anti-cancer or carcinogenic roles in osteosarcoma.

\section{GSEA analysis}

Signed risk scores were divided into high- and lowrisk groups according to the median value, and GSEA 

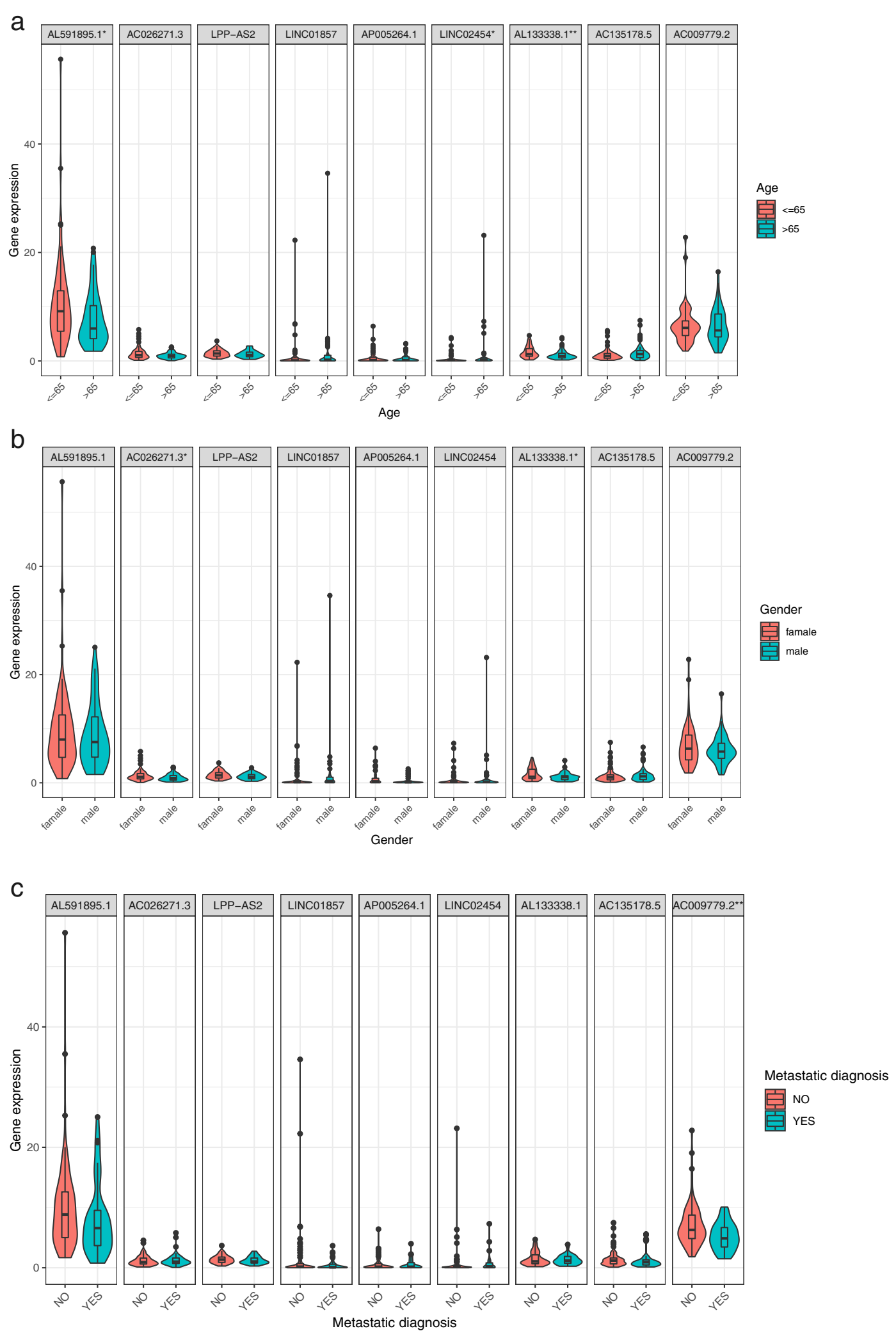

Fig. 5 Correlation analysis of clinical features and individual metabolic IncRNA. Violin diagrams of correlations between (a) age, (b) gender, and (c) metastasis and the expression value of each metabolic IncRNA 

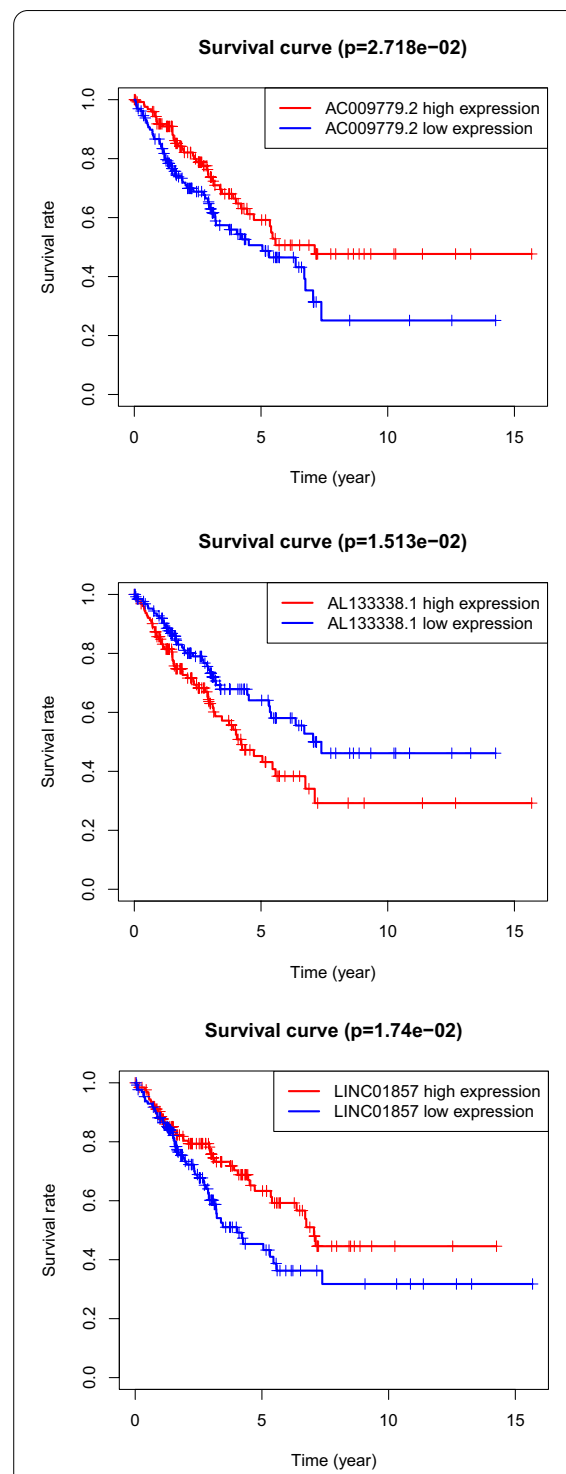
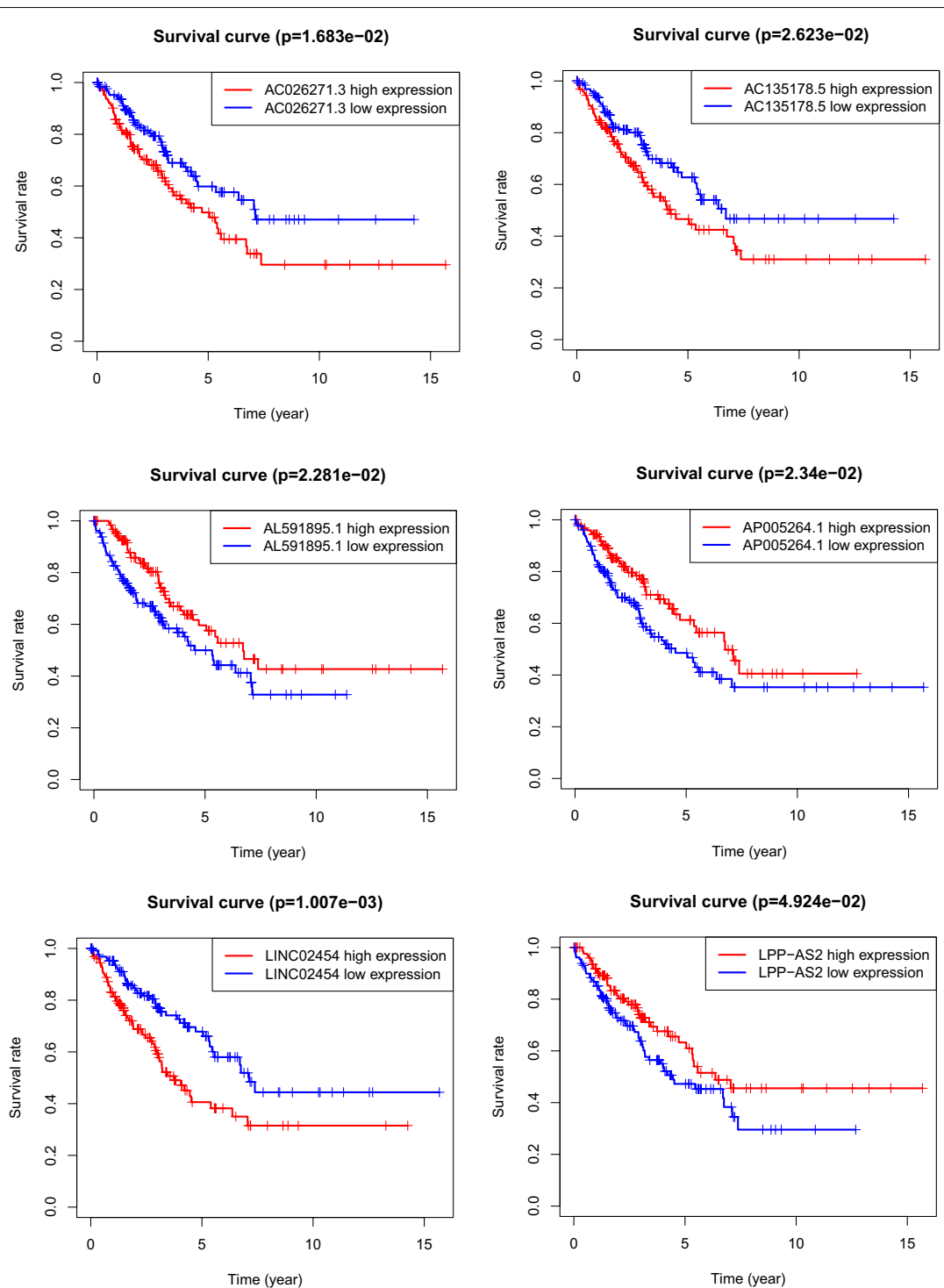

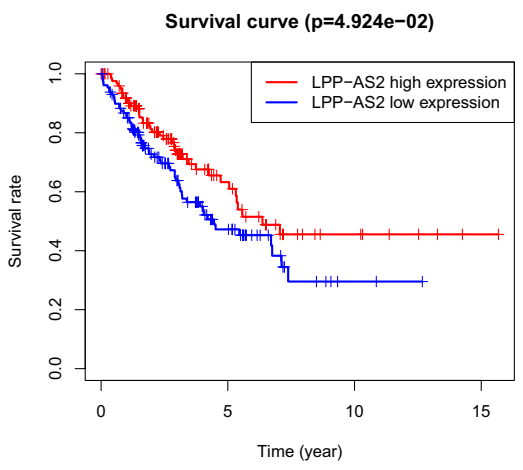

Fig. 6 Survival analysis. The survival curve displayed the different overall survival between high-and low-expression groups of each metabolic InCRNA

analysis was performed on all samples (Fig. 7). In the biological process of gene ontology (GO), we can see the enrichment of multiple biological processes related to bone metabolism. The high-risk groups are significantly enriched in CHONDROCYTE DEVELOPMENT, EMBRYONIC CRANIAL SKELETON MORPHOGENESIS, EMBRYONIC SKELETAL SYSTEM DEVELOPMENT, EMBRYONIC SKELETAL SYSTEM MORPHOGENESIS and HEPARAN SULFATE PROTEOGLYCAN BIOSYNTHETIC PROCESS. The low-risk groups are significantly enriched in the ACTIVATION OF PHOSPHOLIPASE C ACTIVITY, BASE CONVERSION OR SUBSTITUTION EDITING, POLYSACCHARIDE CATABOLIC PROCESS, POLYSACCHARIDE
CATABOLIC PROCESS and REGULATION OF CALCIUM MEDIATED SIGNALING pathways (Fig. 7a). Figure $7 \mathrm{~b}$ and $\mathrm{c}$ reflect the cell component and molecular function of the high- and low-risk groups, respectively. Figure $7 d$ shows the aggregation of metabolic pathways and cancer pathways in the high- and low-risk groups in KEGG. In conclusion, GSEA analysis further describes the potential biological functions of prognostic factors.

\section{Nomogram drawing and PCA}

To make the signature to facilitate clinical prognosis guidance, we drew a nomogram by using available clinical-pathological data and risk score we signed (Fig. 8a). Then, the ROC curve (AUC $=0.839)$ indicates 

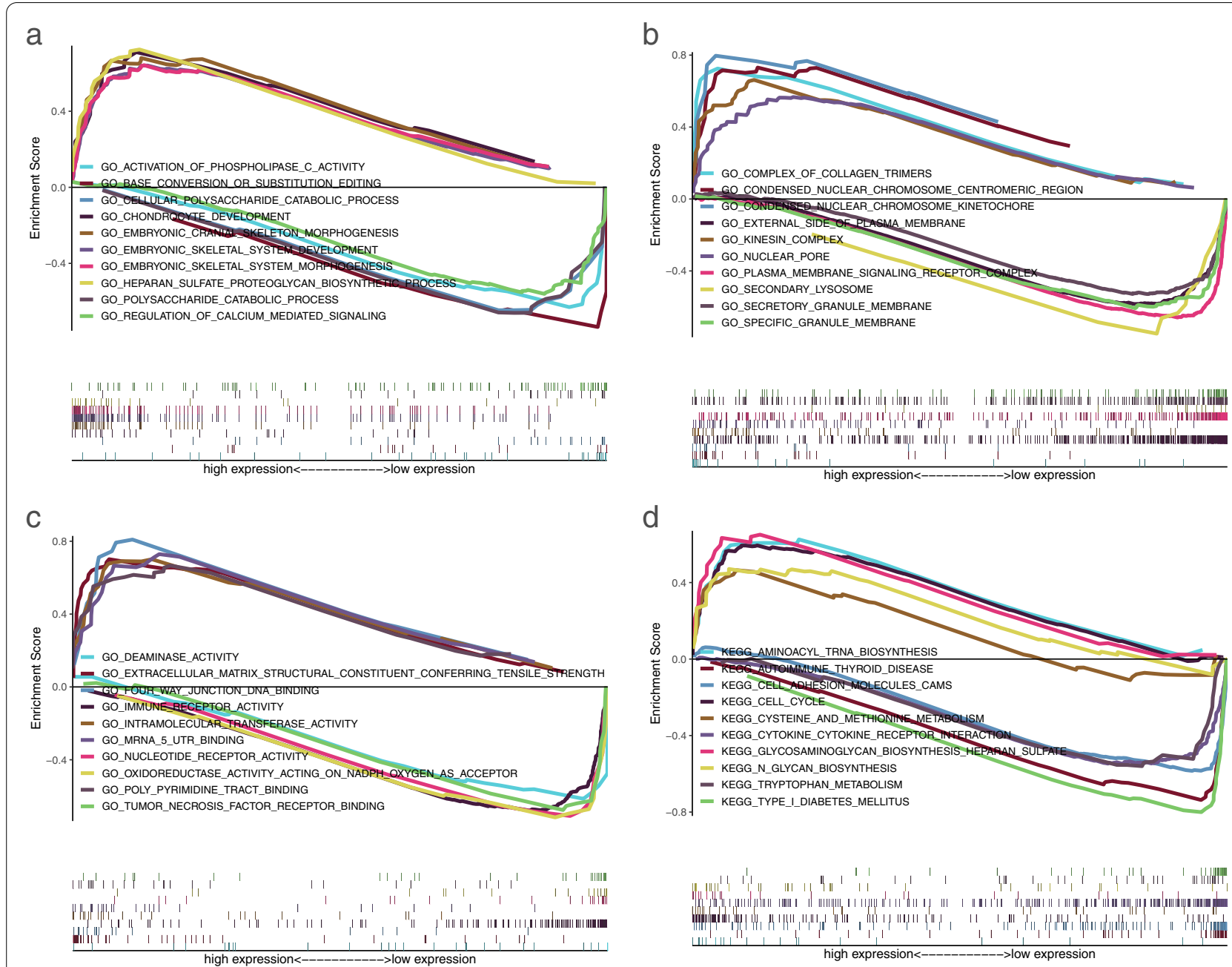

d

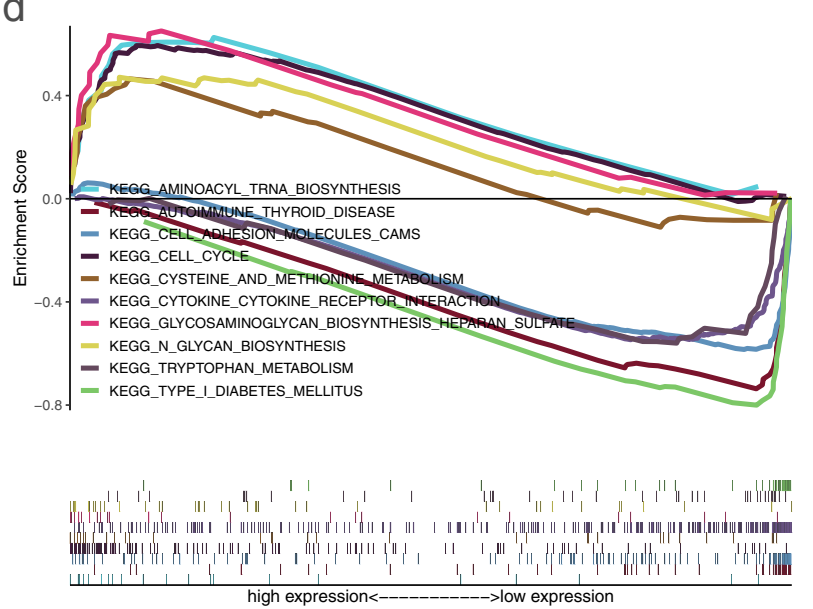

Fig. 7 GSEA analysis. A multi-GSEA plot of the main enrichment pathways of BP (a), CC (b), and MF (c) in GO. A multi-GSEA plot of the main enrichment pathways of KEGG (d)

the nomogram is well representative (Fig. 8b). Further the $C$ index (0.812) establishes that the nomogram has a good distinguishing performance. In addition, we also developed a calibration curve using 1-year, 3 -year, and 5-year survival rates to estimate the accuracy of the nomogram (Fig. 8c). It indicates that the nomogram evaluation of the 3-year survival rate is the most accurate. Finally, to better reflect the difference between the high- and low-risk of each prognostic lncRNA, we conducted a principal component analysis of these prognostic factors. 2d-PCA (Fig. 8d) and 3d-PCA (Fig. 8e) analysis both show that the prognostic signature composed of these IncRNAs can distinguish precisely whether the patient belongs to the low- or high-risk group after dimensionality reduction of the data. Itonce again verifies the accuracy of the signature.

\section{Discussion}

Sarcoma is a malignant tumor derived from connective tissue or other non-epithelial tissues [15]. It occurs in a variety of tissues and organs, including osteosarcoma, leiomyoma, lymphosarcoma, and synovial sarcoma [16], among which osteosarcoma is most common in children and adolescents [17]. Due to the active cartilage and bone metabolism in children and adolescents, osteosarcoma develops rapidly and makes early metastasis [18]. Therefore, the standard of treatment is amputation or chemotherapy [18]. However, $20 \%$ of patients are prone to early metastasis at diagnosis [2], so it is necessary to explore 
a

\section{Points}

age

gender

tumor_depth

tumor_length

tumor_width

Tumor_multifocal

disease recurrence

metastatic_diagnosis

risk_score

Total Points

Linear Predictor

1-year Survival Probability

3-year Survival Probability

5-year Survival Probability

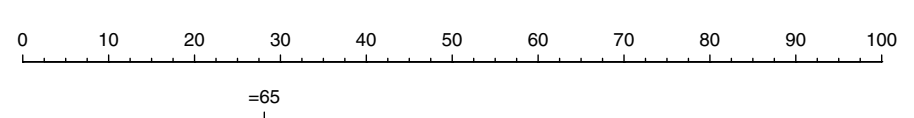

$=65$
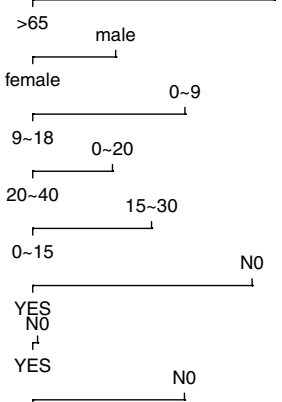

YES

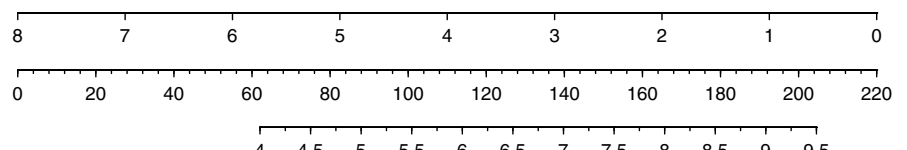

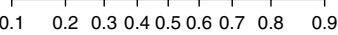

$\begin{array}{lllllllll}0.1 & 0.2 & 0.3 & 0.4 & 0.5 & 0.6 & 0.7 & 0.8 & 0.9\end{array}$

$\begin{array}{lllllllll}0.1 & 0.2 & 0.3 & 0.4 & 0.5 & 0.6 & 0.7 & 0.8 & 0.9\end{array}$ b

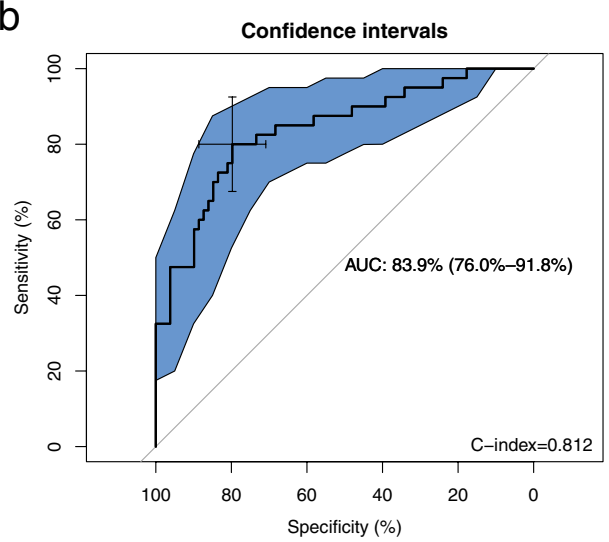

d

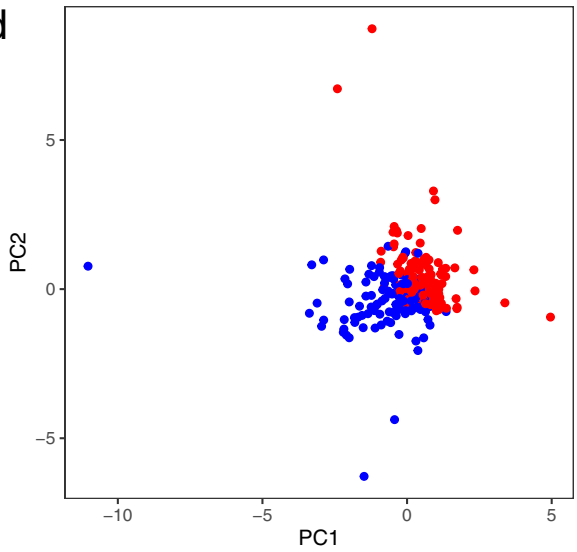

C

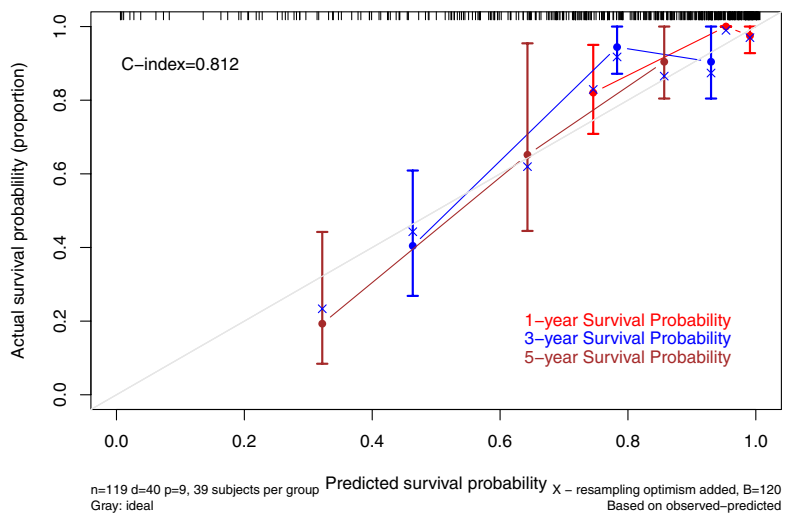

e

- high • low

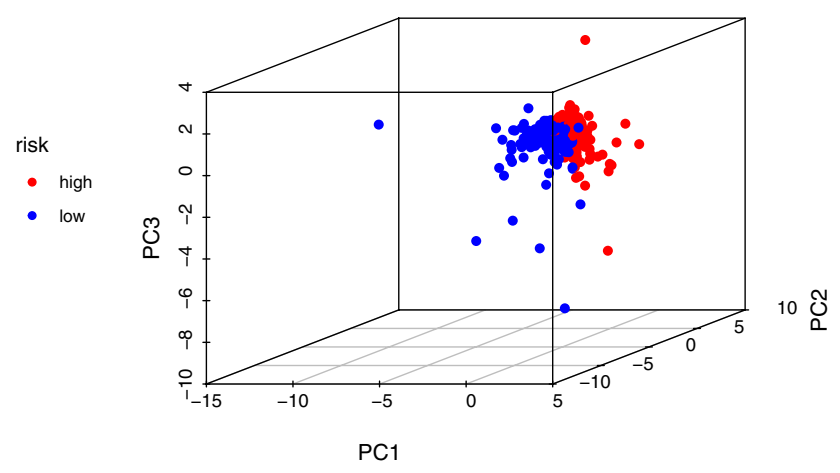

Fig. 8 Drawing the nomogram and principal component analysis. a The nomogram consisted of the risk score and multiple clinical features. b The ROC curve in the nomogram. $\mathbf{c}$ The correction curve in the nomogram. d PCA $2 d$ and (e) PCA $3 d$ 
the pathogenesis and metastasis mechanism. Tumor cells are extremely active in their internal energy and material metabolism due to their immortal proliferation [9]. Several studies have reported the molecular mechanisms about the specific metabolic process of osteosarcoma cells in the body, which proves the key role of the regulation of metabolic pathway in the process of osteosarcoma $[9,19,20]$.

In recent years, with the rapid development of highthroughput technology, the mechanism of various diseases including cancer has been studied more and more deeply, especially in epigenetics, which is represented by lncRNA and circRNA $[4,5]$. Sequencing technology identified five lncRNA types including sense, antisense, bidirectional, intergenic and intronic lncRNAs [21, 22]. These 5 lncRNAs work in different ways. Despite increasing lncRNAs have been reported in osteosarcoma, comparatively few reports reveal roles of lncRNAs in the metabolism of osteosarcoma. Our present study firstly screened out MRlncRNAs in osteosarcoma through comprehensive bioinformatics analysis. It provides guidance for further researches about the molecular mechanism of osteosarcoma metabolism.

In this study, we obtained nine metabolism-related lncRNAs in osteosarcoma, including lncRNA AC009779.2, lncRNA AL591895.1, IncRNA AC026271.3, IncRNA LPP-AS2, IncRNA LINC01857, lncRNA AP005264.1, lncRNA LINC02454, lncRNA AL133338.1 and lncRNA AC135178.5. In addition, we constructed a prognostic signature and the risk score based on the signature. Survival analysis indicates substantially different prognoses between the high- and low-risk groups. The multi-index ROC curve shows that the diagnostic power of the prognostic signature is preferable to clinical factors. At the same time, we also found some lncRNAs were closely related to clinical features such as age, sex and tumor metastasis. All prognostic factors can independently distinguish the prognostic survival rate of patients. GSEA analysis shows that the high- and low-risk groups are closely linked to cancer and multiple metabolic pathways. Finally, we constructed a nomogram to quantify clinical prognosis.

Some of these nine prognostic factors have been reported in other cancers, such as LPP-AS2. LPP-AS2 is thought to be associated with immune cells in soft tissue sarcoma [23]. Another study reported that LPPAS2 regulates EGFR expression by sponging Mir-7-5p as a ceRNA and combination of the promoter region of LPP-AS2 with c-MYC contributes to the development of glioma [24]. LINC01857 has been reported to predict prognosis in hepatocellular carcinoma patients with fibrosis [25]. Another study showed its potential as a novel molecular marker for pancreatic cancer [26]. LINC01857 also can bind to promoters to regulate the transcription process of protein. For example, by interacting with CREBBP to promote the transcription of H3K27Ac and CREB1, LINC01857 promote the progression of breast cancer [27]. In addition, another function of LINC01857 is to combine with miRNAs to play a spongy effect. For example, LINC01857 sponges miR1281 to promote TRIM65 expression and thus facilitates the development of glioma [28]. A recent study revealed that LINC01857 promotes the migration and invasion of gastric cancer cells by regulating microRNA200b [29]. LINC02454, as a long intergenic lncRNA, is mainly reported in papillary thyroid carcinoma $[30,31]$. In addition, other lncRNAs in this study have not been reported in the relevant literature. It suggests that the prognostic signature we constructed is worth in-depth study. Although we integrated a variety of bioinformatics methods and statistical methods, and used various analyses to construct prognostic signatures and multiple verifications, several limitations remain. First, the samples come from a single database, which may be lack of representativeness. Second, no in vivo or in vitro studies were performed. Both the above deficiencies are also the main aspects of our further research.

\section{Conclusion}

We constructed a prognostic signature composed of lncRNAs for the metabolic process of osteosarcoma, which may provide guidance for clinical activities and assist clinical decision-making. In addition, it provided a reference for further study on the regulation of IncRNA on osteosarcoma metabolism.

\section{Abbreviations}

MRIncRNA: Metabolism-related IncRNA; LASSO: Least absolute shrinkage and selection operator; PCA: Principal component analysis; ROC: Receiver operator characteristic; TD: Tumor depth; TL: Tumor length; TW: Tumor width; TM: Tumor multifocal; CREB1: Cyclic AMP-responsive element-binding protein 1; CREBBP: CREB-binding protein.

\section{Supplementary Information}

The online version contains supplementary material available at https://doi. org/10.1186/s13018-021-02647-4.

Additional file 1: Supplementary Table 1. Fifteen Metabolism-related LnCRNA were acquired precisely by Kaplan-Meier survival $(P<0.05)$ analysis and univariable Cox regression survival $(P<0.05)$ analysis.

\section{Acknowledgements}

We would like to acknowledge the TCGA database and GSEA database for free data use.

\section{Authors' contributions}

All authors read and approved the final manuscript. XC designed the study. ZY collected and processed the data. PL and WL conducted in the statistical analyses. XC prepared the manuscript. YL edited the manuscript and provided comments. 


\section{Funding}

This work was no funding supported.

\section{Availability of data and materials}

All data generated or analyzed during this study are included in this published article and its Additional files.

\section{Declarations}

Ethics approval and consent to participate

Not applicable.

\section{Consent for publication}

All authors read and approved the final manuscript.

\section{Competing interests}

The authors declare that they have no competing interests.

\section{Author details}

'Spinal Surgery, The First People's Hospital of Jingmen, Jingmen, Hubei, China. ${ }^{2}$ Department of Gastroenterology, The First People's Hospital of Jingmen, Xiangshan Avenue 168, Jingmen 448000, Hubei, China.

Received: 1 May 2021 Accepted: 29 July 2021

Published online: 23 August 2021

\section{References}

1. Ritter J, Bielack SS. Osteosarcoma. Ann Oncol. 2010;21(Suppl 7):vii320-5.

2. Rainusso N, Wang LL, Yustein JT. The adolescent and young adult with cancer. state of the art -- bone tumors. Curr Oncol Rep. 2013;15(4):296-307.

3. Dean DC, Shen S, Hornicek FJ, Duan Z. From genomics to metabolomics emerging metastatic biomarkers in osteosarcoma. Cancer Metastasis Rev. 2018;37(4):719-31.

4. Tano K, Akimitsu N. Long non-coding RNAs in cancer progression. Front Genet. 2012;3:219.

5. Shi X, Sun M, Liu H, Yao Y, Song Y. Long non-coding RNAs a new frontier in the study of human diseases. Cancer Lett. 2013;339(2):159-66.

6. Bhan A, Soleimani M, Mandal SS. Long Noncoding RNA and cancer a new paradigm. Cancer Res. 2017;77(15):3965-81.

7. Li Z, Yu X, Shen J. Long non-coding RNAs emerging players in osteosarcoma. Tumour Biol. 2016;37(3):2811-6.

8. Taniguchi M, Fujiwara K, Nakai Y, Ozaki T, Koshikawa N, Toshio K, Kataba M, Oguni A, Matsuda H, Yoshida Y, et al. Inhibition of malignant phenotypes of human osteosarcoma cells by a gene silencer, a pyrrole-imidazole polyamide, which targets an E-box motif. FEBS Open Bio. 2014;4:328-34.

9. Shen S, Yao T, Xu Y, Zhang D, Fan S, Ma J. CircECE1 activates energy metabolism in osteosarcoma by stabilizing c-Myc. Mol Cancer. 2020;19(1):151.

10. Chen $Y$, Zhao B, Wang $X$. Tumor infiltrating immune cells (TIICS) as a biomarker for prognosis benefits in patients with osteosarcoma. BMC Cancer. 2020;20(1):1022.

11. Niu J, Yan T, Guo W, Wang W, Zhao Z, Ren T, Huang Y, Zhang H, Yu Y, Liang $X$. Identification of Potential Therapeutic Targets and Immune Cell Infiltration Characteristics in Osteosarcoma Using Bioinformatics Strategy. Front Oncol. 2020;10:1628.

12. Shen $Y, X u$ J, Pan $X$, Zhang $Y$, Weng $Y$, Zhou D, He S. LncRNA KCNQ1OT1 sponges miR-34c-5p to promote osteosarcoma growth via ALDOA enhanced aerobic glycolysis. Cell Death Dis. 2020;11(4):278.

13. The Cancer Genome Atlas (TCGA) database. (https://gdc-portal.nci.nih. gov/). Accessed 3 Oct 2020.
14. Subramanian A, Tamayo P, Mootha VK, Mukherjee S, Ebert BL, Gillette MA, Paulovich A, Pomeroy SL, Golub TR, Lander ES, et al. Gene set enrichment analysis a knowledge-based approach for interpreting genome-wide expression profiles. Proc Natl Acad Sci USA. 2005;102(43):15545-50.

15. Blay J-Y, Ray-Coquard I. Sarcoma in 2016. Evolving biological understanding and treatment of sarcomas. Nat Rev Clin Oncol. 2017;14(2):78-80.

16. Pappo AS, Dirksen U. Rhabdomyosarcoma, Ewing sarcoma, and other round cell sarcomas. J Clin Oncol. 2018;36(2):168-79.

17. Kager L, Tamamyan G, Bielack S. Novel insights and therapeutic interventions for pediatric osteosarcoma. Future Oncol. 2017;13(4):357-68.

18. Bielack SS, Kempf-Bielack B, Delling G, Exner GU, Flege S, Helmke K, Kotz R, Salzer-Kuntschik M, Werner M, Winkelmann W, et al. Prognostic factors in high-grade osteosarcoma of the extremities or trunk an analysis of 1,702 patients treated on neoadjuvant cooperative osteosarcoma study group protocols. J Clin Oncol. 2002;20(3):776-90.

19. Nakajima K, Raz A. Autocrine motility factor and its receptor expression in musculoskeletal tumors. J Bone Oncol. 2020;24:100318.

20. Yang C, Tian Y, Zhao F, Chen Z, Su P, Li Y, Qian A. Bone Microenvironment and Osteosarcoma Metastasis. Int J Mol Sci. 2020;21(19):6985.

21. Kopp F, Mendell JT. Functional classification and experimental dissection of long noncoding RNAs. Cell. 2018;172(3):393-407.

22. St Laurent $G$, Wahlestedt $C$, Kapranov P. The landscape of long noncoding RNA classification. Trends Genet. 2015;31(5):239-51.

23. Huang R, Meng T, Chen R, Yan P, Zhang J, Hu P, Zhu X, Yin H, Song D, Huang $Z$. The construction and analysis of tumor-infiltrating immune cell and ceRNA networks in recurrent soft tissue sarcoma. Aging (Albany NY). 2019;11(22):10116-43.

24. Zhang X, Niu W, Mu M, Hu S, Niu C. Long non-coding RNA LPP-AS2 promotes glioma tumorigenesis via miR-7-5p/EGFR/PI3K/AKT/c-MYC feedback loop. J Exp Clin Cancer Res. 2020;39(1):196.

25. Ye J, Wu S, Pan S, Huang J, Ge L. Risk scoring based on expression of long non-coding RNAs can effectively predict survival in hepatocellular carcinoma patients with or without fibrosis. Oncol Rep. 2020;43(5):1451-66.

26. Giulietti M, Righetti A, Principato G, Piva F. LncRNA co-expression network analysis reveals novel biomarkers for pancreatic cancer. Carcinogenesis. 2018;39(8):1016-25.

27. Xiong Y, Gu Y, Wang F, Li L, Zhu M, Wang N, Mi H, Qiu X. LINC01857 as an oncogene regulates CREB1 activation by interacting with CREBBP in breast cancer. J Cell Physiol. 2019;234(8):14031-9.

28. Hu G, Liu N, Wang H, Wang Y, Guo Z. LncRNA LINC01857 promotes growth, migration, and invasion of glioma by modulating miR-1281/ TRIM65 axis. J Cell Physiol. 2019;234(12):22009-16.

29. Chen GR, Sun W, Zheng K, Zhu W. LINC01857 promotes the development of gastric cancer by regulating microRNA-200b. Eur Rev Med Pharmacol Sci. 2020;24(7):3648-56.

30. Cai W-Y, Chen X, Chen L-P, Li Q, Du X-J, Zhou Y-Y. Role of differentially expressed genes and long non-coding RNAs in papillary thyroid carcinoma diagnosis, progression, and prognosis. J Cell Biochem. 2018;119(10):8249-59.

31. Tan J, Liu L, Zuo Z, Song B, Cai T, Ding D, Lu Y, Ye X. Overexpression of novel long intergenic non-coding RNA LINC02454 is associated with a poor prognosis in papillary thyroid cancer. Oncol Rep. 2020;44(4):1489-501.

\section{Publisher's Note}

Springer Nature remains neutral with regard to jurisdictional claims in published maps and institutional affiliations. 